Review Article

\title{
Mitochondrial Nucleoid: Shield and Switch of the Mitochondrial Genome
}

\author{
Sung Ryul Lee ${ }^{1}$ and Jin Han ${ }^{2}$ \\ ${ }^{1}$ Department of Integrated Biomedical Science, Cardiovascular and Metabolic Disease Center, College of Medicine, Inje University, \\ Busan, Republic of Korea \\ ${ }^{2}$ National Research Laboratory for Mitochondrial Signaling, Department of Physiology, Cardiovascular and Metabolic Disease \\ Center, College of Medicine, Inje University, Busan, Republic of Korea
}

Correspondence should be addressed to Jin Han; phyhanj@inje.ac.kr

Received 18 January 2017; Revised 6 March 2017; Accepted 3 April 2017; Published 7 June 2017

Academic Editor: Maik Hüttemann

Copyright (c) 2017 Sung Ryul Lee and Jin Han. This is an open access article distributed under the Creative Commons Attribution License, which permits unrestricted use, distribution, and reproduction in any medium, provided the original work is properly cited.

\begin{abstract}
Mitochondria preserve very complex and distinctively unique machinery to maintain and express the content of mitochondrial DNA (mtDNA). Similar to chromosomes, mtDNA is packaged into discrete mtDNA-protein complexes referred to as a nucleoid. In addition to its role as a mtDNA shield, over 50 nucleoid-associated proteins play roles in mtDNA maintenance and gene expression through either temporary or permanent association with mtDNA or other nucleoid-associated proteins. The number of $\operatorname{mtDNA}(\mathrm{s})$ contained within a single nucleoid is a fundamental question but remains a somewhat controversial issue. Disturbance in nucleoid components and mutations in mtDNA were identified as significant in various diseases, including carcinogenesis. Significant interest in the nucleoid structure and its regulation has been stimulated in relation to mitochondrial diseases, which encompass diseases in multicellular organisms and are associated with accumulation of numerous mutations in mtDNA. In this review, mitochondrial nucleoid structure, nucleoid-associated proteins, and their regulatory roles in mitochondrial metabolism are briefly addressed to provide an overview of the emerging research field involving mitochondrial biology.
\end{abstract}

\section{Introduction}

Normal cellular physiology is critically dependent upon energy in eukaryotic cells, making mitochondria indispensable organelles for energy production in the form of adenosine triphosphate (ATP) via the electron-transport chain and oxidative phosphorylation system (OXPHOS). Additionally, numerous biological functions, including ATP transport, heat production, metal homeostasis, and stress signaling and defense responses, involve mitochondria [1-5]. Stationary (or immobilized) mitochondria serve as calcium buffers to avoid harmful intracellular calcium overload. Depending on cellular demand, their composition is highly variable from tissue to tissue to enable fulfillment of specialized functions, with accumulation at regions of high-energy demand $[4,6]$. The position of mitochondria within the cell is determined largely by the cytoskeleton, which comprises a highly dynamic network of actin filaments, microtubules, and intermediate filaments $[7,8]$. Mitochondrial movement, which appears to be influenced by intermediate filament proteins, is highly coordinated with changes in organelle shape in order to produce mitochondria with sizes compatible with their movement [9]. Therefore, the correct distribution of mitochondria is achieved by directed movement and docking and anchoring mechanisms [8]. Unlike other subcellular organelles, such as Golgi, lysosomes, and endosomes, mitochondria individually encapsulate their own genome, referred to as mitochondrial DNA (mtDNA). The size range of mtDNAs found in multicellular animals is relatively narrow ( 16.5 kb; Figure 1), with some exceptions varying from $14 \mathrm{~kb}$ in the nematode to $42 \mathrm{~kb}$ in the scallop [10]. However, the mitochondrial genome of higher plants is much larger than that in multicellular animals, ranging from $200 \mathrm{~kb}$ to $2400 \mathrm{~kb}[6,10]$. Many aspects of mtDNA differ 


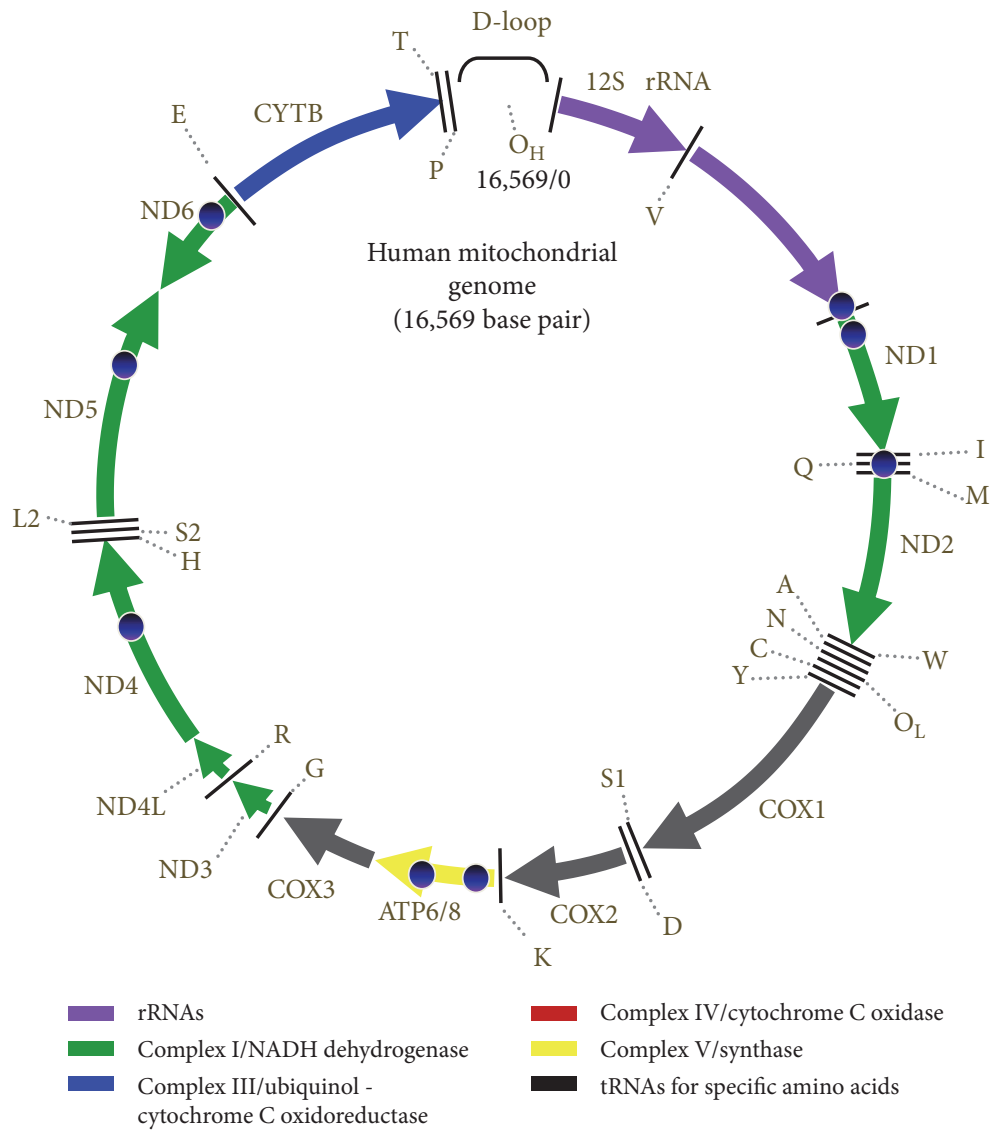

Figure 1: Human mtDNA. Human mtDNA is circular, with 16,569 bps that encode seven of the 43 subunits of complex I, one of the 11 subunits of complex III (CYTB), three of the 13 subunits of complex IV (COXI, COXII, and COXIII), and two of the 16 subunits of complex V (ATP synthase 6 and ATP synthase 8). It also encodes two ribosomal RNAs and 22 transfer RNAs. ATP, adenosine triphosphate; COX, cyclooxygenase; CYTB, cytochrome B; mtDNA, mitochondrial DNA.

from those of nuclear DNA, including its non-Mendelian genetics and the polyploid nature of the genome within a single cell $[11,12]$.

Mitochondria preserve very complex and unique machinery to maintain and express the content of mtDNA. For example, mtDNA replication occurs independent of the cell cycle and irrespective of the replication of genes in the nucleus [13]. Mutations originating from chromosomal DNA cannot completely explain mitochondrial diseases manifested in cardiomyopathies [14, 15], neurodegenerative diseases, aging [16-18], and cancer. Mitochondrial genomes are not naked but rather packaged into chromosome-like organellar nuclei, termed nucleoids, that exhibit a discrete macromolecular assembly that dictates mtDNA-protein interactions related to mitochondrial genetics [19]. In eukaryotic cells, thousands of mtDNA molecules are organized into several hundred nucleoids [1, 13, 19-24], which function as units of $\mathrm{mtDNA}$ propagation for $\mathrm{mtDNA}$ replication, segregation, and gene expression [25-28]. As an organizing body of mtDNA, nucleoids work as a platform for the subtle and controlled regulation of mitochondrial genomes and their efficient integration into cellular signaling $[26,29]$. Naked mtDNA in the mitochondrial matrix would preclude efficient mtDNA maintenance, resulting in increased accumulation of mutations and the inevitable faulty segregation of mtDNA. Numerous cellular metabolic processes are connected to dynamic regulation associated with mitochondrial nucleoids in order to control the stabilization, maintenance, distribution, and inheritance of the mitochondrial genome [30, 31]. In this review, we addressed the putative mitochondrial nucleoid structure, proteins involved in nucleoid formation, and their regulatory roles in mitochondrial metabolism. Although in-depth mechanistic findings regarding mtDNA nucleoids have been extensively revealed in model organisms, such as Saccharomyces cerevisiae [32], this review will be limited to findings from human and mammalian systems.

\section{Mitochondrial Structure and Shape}

Mitochondrial morphology, suggested as ovoid or rodshaped, is not static, and mitochondria have no fixed size but vary in appearance of the cristae and structure, which can be branched, curved, or elongated and rod-like and fragmented into multiple smaller mitochondria depending on cell type [33-35]. Even within individual cells, mitochondrial structure varies. For example, mitochondria in skeletal muscle are ovoid structures, with two possible populations: one positioned close to the sarcolemma and the other embedded among the myofibrils [33]. In unstressed or 


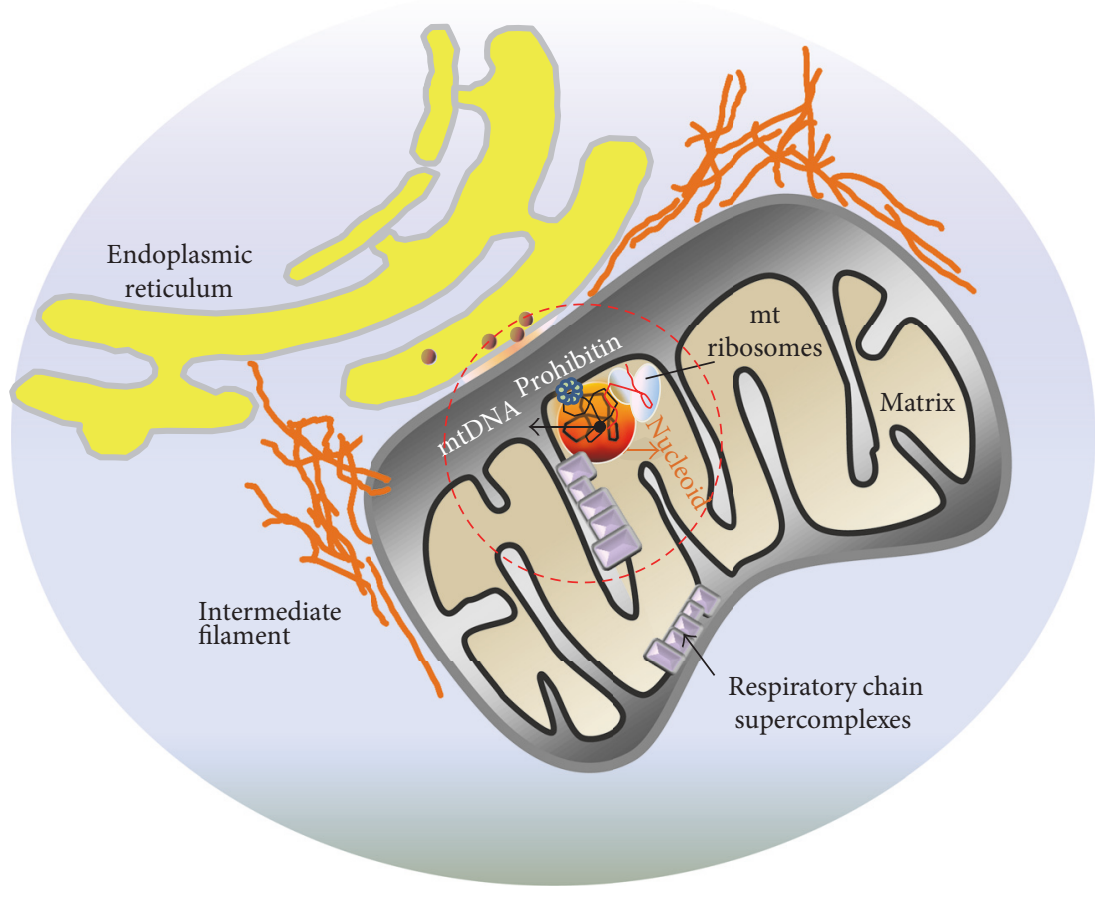

FIGURE 2: Spatial organization (localization) of mitochondrion and mitochondrial nucleoids. The contour length of circular mtDNA $(\sim 16.5 \mathrm{~kb})$ is $\sim 5 \mu \mathrm{m}$ [47] and requires tight packaging into nucleoids to fit into the tubules of the mitochondrial network comprised of cylinders $\sim 250 \mathrm{~nm}$ to $\sim 400 \mathrm{~nm}$ in diameter. Nucleoids are organized in higher-ordered assemblies, including respiratory chain supercomplexes [31] and ER-mitochondria complexes (red circle). ER, endoplasmic reticulum; mtDNA, mitochondrial DNA.

intact nondividing cells, mitochondria exist not as a separate, individual mitochondrion, which is routinely seen in isolated mitochondria as a fractional artifact, but rather as a highly connected reticular network. This reticular network of mitochondria is influenced by fission and fusion executed by mitochondria-shaping proteins called mitodynamins [34]. Fission can be initiated through the response of mitodynamins to mitochondrial energetics, oxidative stress, hypoxia, or mtDNA damage [34]. When a daughter mitochondrion depolarizes following a fission event and is unable to fuse to the reticular network, this solitary mitochondrion will be removed by mitophagy [36]. Structurally, mitochondria exhibit a double-membrane arrangement, which separates the organelle into four distinct compartments (Figure 2): the outer membrane, the intermembrane space, the inner membrane, and the matrix [33, 37]. The outer membrane separates mitochondria from the cytoplasm and surrounds the inner membrane, which separates the intermembrane space from the protein-dense central matrix. Unlike the outer membrane, the inner membrane constitutes a tight diffusion barrier against all ions and molecules and is differentiated into the inner boundary membrane and the cristae, which is the site of mtDNA replication, transcription, protein biosynthesis, and numerous enzymatic reactions. The two regions are continuous at cristae junctions, with cristae extending into the matrix and acting as the primary sites of mitochondrial energy conversion by ATP synthase located in cristae membranes [37]. Mitochondria do not float freely in the cytosol but are positioned in the cytosol with the aid of intermediate filaments and likely through molecular linkages, networks, and bidirectional signaling between xellular components and intermediate filaments [7, 8]. Mitochondrial dynamics are responsible for intracellular distribution and reactions based on functional requirements that are maintained through fission, fusion, growth, and structural reorganization, followed by turnover and rearrangements of mitochondrial proteins and DNA [33, 38-40]. Nucleoid foci containing mtDNA are attached to the cytoskeleton [7] and organize the translation machinery on both sides of the mitochondrial membranes [7]. In view of the organization of general mitochondrial functions (Figure 2), many processes are organized in higher-ordered assemblies, including the respiratory chain supercomplexes [41], endoplasmic reticulum(ER-) mitochondria complexes [42, 43] involved in organelle biogenesis and inheritance, mitochondrial-contact sites and cristae-organizing-system complexes [44] responsible for the organization of mitochondrial ultrastructure and biogenesis, and mitochondrial membrane supercomplexes that mediate protein trafficking $[45,46]$. The detailed description of these complexes is under investigation and is beyond the scope of this review.

\section{Mitochondrial DNA}

Human mtDNA consists of circular, double-stranded 16,569 bp DNA with a contour length of $\sim 5 \mu \mathrm{m}$ [47], thus requiring mtDNA to be highly packaged to fit into a $\sim 100 \mathrm{~nm}$ (in diameter) nucleoid [37]. A mitochondrion contains at least 800 to 1500 proteins of varying relative abundance between tissues [48]; however, mtDNA contains only 37 genes $[49,50]$ encoding 13 proteins of the mitochondrial respiratory chain, two ribosomal RNAs (12S and 16S), 
and 22 transfer RNAs (Figure 1). The remaining protein subunits that comprise the OXPHOS, together with those required for mtDNA maintenance, are encoded by nuclear DNA, synthesized by cytoplasmic ribosomes, and are specifically targeted and sorted into their correct locations within the mitochondrion. Unlike nuclear DNA, mtDNA is characterized by high gene density and the absence of introns [51]. With the exception of a $\sim 1 \mathrm{~kb}$ noncoding regulatory fragment (D-loop), mtDNA is entirely transcribed as large polycistrons from both strands [51]. Technically, mtDNA in the nucleoid can be localized in fixed cells in two ways: immunolabeling using an anti-DNA antibody or cell growth for one generation in bromodeoxyuridine (BrdU) to uniformly label the DNA to enable detection using an anti-BrdU antibody [7]. Additionally, mtDNA can be stained with 4',6-diamidino-2phenylindole, ethidium bromide, or PicoGreen dye [52]. Despite mtDNA being essential for normal physiological functions, the genome is vulnerable to oxidative stress [53]. When isolated rat cardiomyocytes were treated with $50 \mu \mathrm{M}$ $\mathrm{H}_{2} \mathrm{O}_{2}$, the amount of intact $16 \mathrm{kbp}$ mtDNA decreased by $50 \%$ over $10 \mathrm{~min}$, resulting in oxidative stress and leading to mitochondrial dysfunction due to the decline in the activities of complexes I, III, and IV, all of which contain mtDNAencoded subunits [54]. mtDNA constantly undergoes mutation, with clonal expansion or loss of either point mutations or deletions [12], and mutations of mtDNA, both point mutations and deletions, cause a host of tissue-specific [15] and systemic diseases $[12,55]$. The polyploid nature of the mitochondrial genome (up to several thousand copies per cell) gives rise to the important features of homoplasmy, heteroplasmy [56-59], and clonal expansion of mtDNA, even in the same mitochondrion, with random mitochondrial segregations capable of occurring in mitochondria within the same cell $[11,12]$. Based on the presence of heteroplasmy and clonal segregation, mtDNA status, regardless of harbored mutations, may not be an important factor in the construction and maintenance of nucleoid organization, given that nucleoid-containing mutated mtDNA can segregate in the cell. Although additional study is required to understand the behavior of nucleoid-containing mutated mtDNA and its propagation, why and how mitochondria (or cells) tolerate this aberrant condition remain an interesting question.

\section{Mitochondrial Nucleoid Structure and Dynamics}

The term nucleoid was first used in 1937 by Piekarski to describe the envelope-lacking structure of the bacterial chromosome as being distinct from that of eukaryotes [60]. Similar DNA-containing structures were later discovered in mitochondria and plastids [61]. Nucleoids do not contain membranes capable of separating the nucleoid compartment from the matrix [27]. The mitochondrial nucleoid is composed of mtDNA and numerous nucleoid-associated proteins (Figure 2) that form a macromolecular structure capable of providing submitochondrial organization of mtDNA [29, 62]. Efficient maintenance of mtDNA in discrete, segregated units located at intervals throughout the mitochondrial network is concerted through the control of nucleoid structure [32].

The organization of the nucleoid is a very fundamental question in mitochondrial biology. The crucial structural difference between nuclear chromatin and mitochondrial nucleoid is that mtDNA is not associated with histones in the form of nucleosomes [20,63]. Nucleoids are roughly spherical, with a diameter of $\sim 100 \mathrm{~nm}$ and with each containing more than one copy of mtDNA [37]. In view of their size, nucleoids must fit into the $\sim 10 \mu \mathrm{m}$ tubules of the mitochondrial tubular network, which can be approximated by infinite cylinders of $\sim 250 \mathrm{~nm}$ to $\sim 400 \mathrm{~nm}$ diameter $[64,65]$. In human cells, the multilayer model of mitochondrial nucleoid organization [66], which describes separation into the inner core region where DNA and proteins (DNA-packaging proteins and proteins involved in replication and transcription) are concentrated and the outer peripheral region containing proteins temporarily recruited to execute special functions in the nucleoid, was suggested based on the tightly bound mtDNA [35, 61]. mtDNA replication and transcription occur in the core region through the activity of mitochondrial transcription factor A (TFAM), mitochondrial RNA polymerase (POLRMT), mitochondrial single-stranded DNA-binding protein (mtSSB), mitochondrial polymerase $\gamma$ (POLG), and Twinkle helicase, with subsequent RNA processing and translation occurring in the outer region (peripheral region) $[22,61,67]$. In the peripheral region of nucleoids, numerous putative proteins were also identified [61]; however, less is known concerning nucleoid states during mtDNA replication and/or transcription. As shown in Figure 3, mtDNA can be compactly packaged by the binding of mitochondrial transcription factor A (TFAM). The D-loop region of mtDNA, which constitutes a regulatory site for mtDNA replication and transcription, is anchored to the inner mitochondrial membrane (Figures 2 and 3) likely through a multiprotein complex $[41,42,44]$ and serves as a central hub for forming nucleoids. As depicted in Figure 4, POLG, ATPase family AAA-domain-containing protein 3 (ATAD3), and the mitochondrial AAA proteases Lon peptidase 1 (LONP1) and mtSSB, including TFAM, are believed to be nucleoid-associated proteins that might interact with the D-loop region of mtDNA $[22,62,68]$. These mtDNAbinding proteins are involved in interactions between mtDNA and the mitochondrial inner membrane, ribosome, and other supercomplexes to facilitate transport of proteins or molecules [45, 69-71]. Mutations in the Dloop region might result in altered binding affinities for the nuclear proteins involved in mtDNA replication and transcription, resulting in severe depletion of mtDNA content due to replication failure and disruption of nucleoid structure $[12,32,72-75]$.

\section{Nucleoid Structure and Dynamics}

Nucleoid structure may vary between tissue types and according to age [76]. Nucleoids are tethered directly or indirectly via the mitochondrial membrane to kinesin and microtubules in the surrounding cytoplasm [7]. Additionally, nucleoids are composed of thin filaments that protrude 


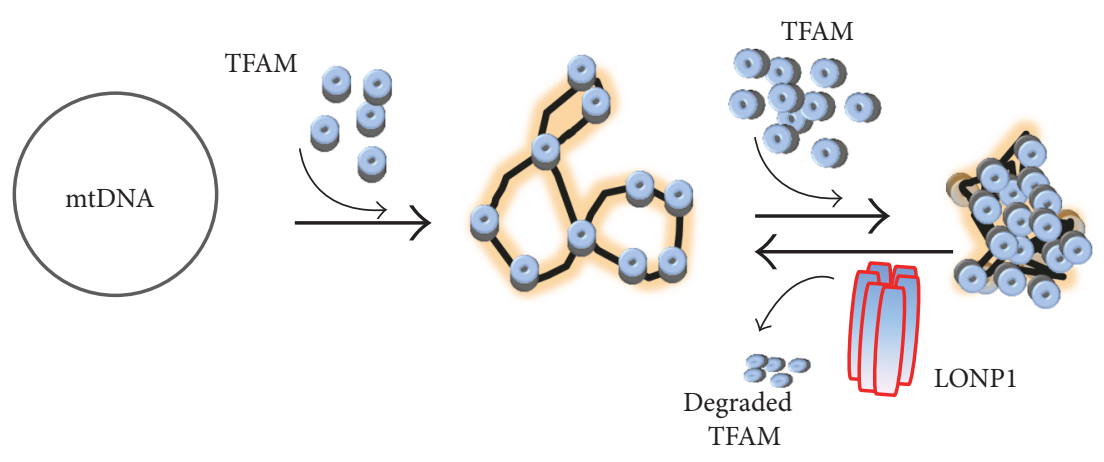

FIgURE 3: mtDNA packaging by TFAM and its degradation by the mitochondrial AAA protease LONP1. Human circular mtDNA is packaged by TFAMs, but their excessive packing of mtDNA may result in shutdown of mtDNA transcription and replication. TFAM can be degraded by the mitochondrial protease LONP1. LonP1, mitochondrial AAA protease; mtDNA, mitochondrial DNA; TFAM, mitochondrial transcription factor A.

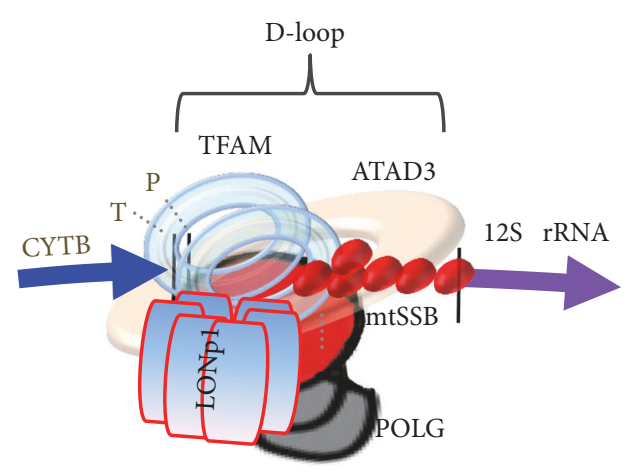

FIGURE 4: Putative nucleoid-associated proteins located in the Dloop region of mtDNA. TFAM, POLG, ATAD3, LONP1, and $\mathrm{mtSSB}$ are nucleoid-associated proteins that possibly interact with the D-loop region of mtDNA. These five proteins might exhibit DNA-binding capacity and, therefore, directly associate with mtDNA. ATAD3, ATPase family AAA-domain-containing protein 3; LONP1, mitochondrial AAA protease; mtDNA, mitochondrial DNA; mtSSB mitochondrial single-stranded DNAbinding protein; POLG, mitochondrial polymerase $\gamma$; TAFM, mitochondrial transcription factor A.

outward and serve as anchors to the membrane [21]. Movement of nucleoids located in the protein-dense matrix compartment is limited due to their attachment to the mitochondrial inner membrane (Figure 5), which precludes free diffusion through the matrix compartment [7]. A subset of nucleoids can be observed in close proximity to microtubules, which are used to transport mitochondria over long distances and suggest important roles for the cytoskeleton in nucleoid movement, division, and/or sorting [63]. Nucleoids containing nascent mtDNA localize to mitochondrial tips, with these products of division preferentially distributed within cells as compared with nonreplicative nucleoids [70]. In addition, nucleoids actively engaged in mtDNA synthesis in mammalian cells are spatially and temporally linked to a small subset of ER-mitochondria contacts destined for mitochondrial division [37].

During mtDNA transcription or replication, numerous nucleoid-associated proteins are recruited, indicating that the mitochondrial nucleoid is dynamic and not a single discrete entity $[7,77]$. The kinetics of replication and transcription (monitored by immunolabeling after incorporation of BrdU or bromouridine) suggest that each mtDNA replicates independently of others and that newly made RNA remains (resident half-life: $\sim 43 \mathrm{~min}$ ) long after it has been made [7]. During transcription or replication, nucleoids should be relaxed to facilitate attachment of transcription factors; therefore, the size of such active nucleoids might be larger than that of quiescent nucleoids due to the surrounding shell of proteins associated with the replication and transcription machinery [64]. Unlike a single nucleoid, nucleoid clusters will be formed and mostly contain nucleoids surrounding newly replicated mtDNA; however, the nucleoid population not in replication mode remains outside of these clusters [78]. It was suggested that mitochondrial nucleoids can be reversibly clustered with the aid of TFAM upon mtDNA stress and that this nucleoid clustering might be beneficial for newly replicated mtDNA against intercalators, such as the mtDNA-depletion agent ethidium bromide or anticancer drugs [78].

Within the mitochondrion, nucleoids show an asymmetric intracellular distribution determined by mitochondrial division, fusion, and motility events [70], suggesting that the nucleoid-transmission process is DNA-independent and reliant upon protein-protein interactions [63]. At division sites, mtDNA replication occurs upstream of mitochondrial constriction and assembly of the division machinery. In the absence of mtDNA leading to defects in respiratory activity and energy production, nucleoid integrity is lost due to the absence of protein-DNA and additional protein-protein interactions, and the mitochondrial reticulum is compromised due to the reduced cristal-membrane content [79]. However, nucleoid proteins are capable of binding to their proper sites on the inner mitochondrial membrane and are sorted normally in the absence of mtDNA, given the nearuniform distribution of $\mathrm{mtSSB}$ in mtDNA-depleted rhozero cells [80]. The maintenance or selective degradation of mitochondrial nucleoids free of mtDNA remains unknown.

In mammalian cells, mtDNA exhibits a closed, circular form; however, upon strand breakage or partial deletion, this circular structure is linearized. This linearized and unsealed 


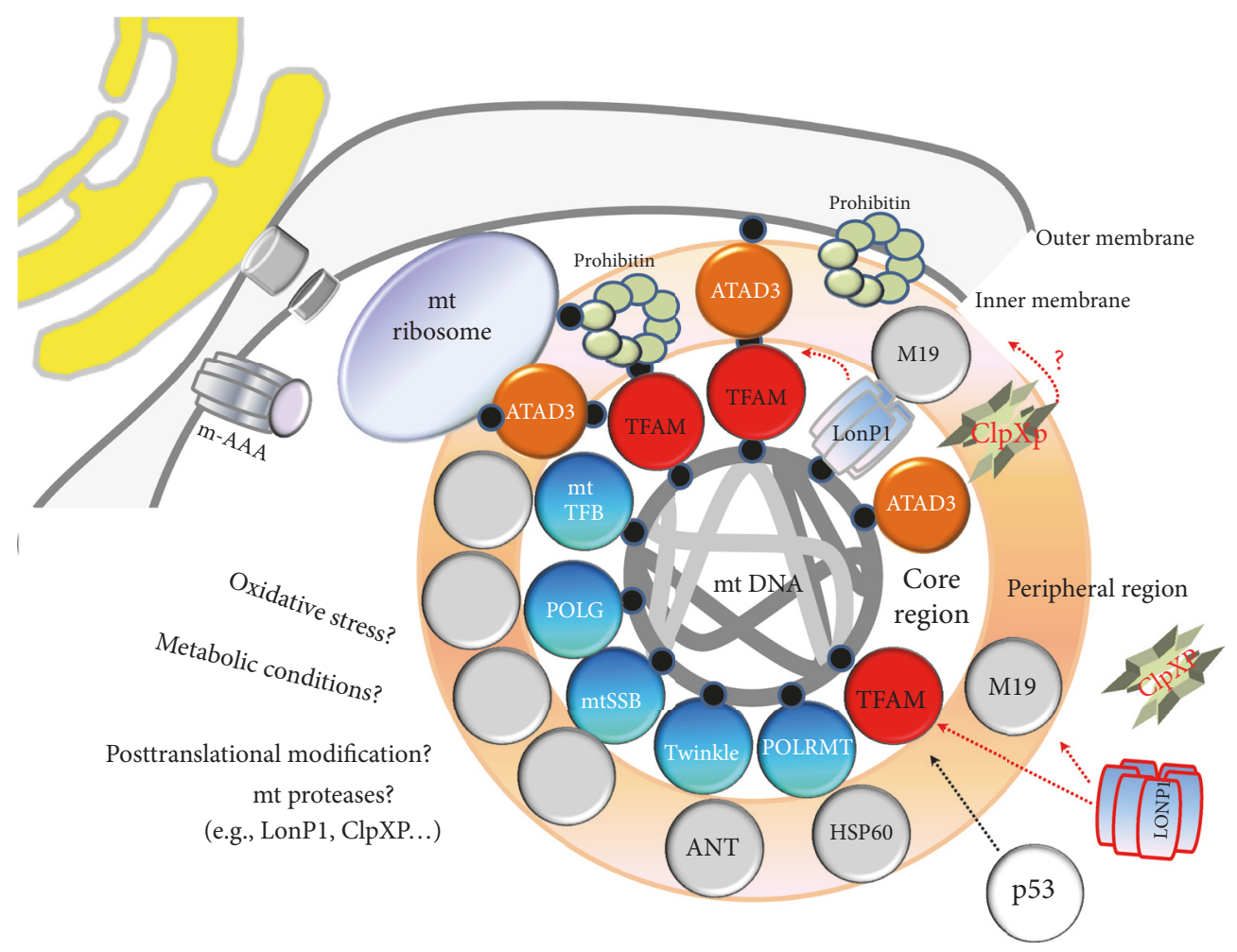

FIGURE 5: Spatial organization of nucleoid-associated proteins. Some proteins that exhibit mtDNA-binding capacity are located in the core region, whereas others might interact with proteins in the core region or other mitochondrial proteins. ANT, adenine nucleotide translocator; ATAD3, ATPase family AAA-domain-containing protein 3; HSP60, heat-shock protein 60; LONP1, mitochondrial AAA protease; $\mathrm{mAAA}$, mitochondrial ATPase-associated with various cellular activities (AAA+) protease; mtDNA; mitochondrial DNA; mtSSB, mitochondrial single-stranded DNA-binding protein; mTERF, mitochondrial transcription termination factor; PHB; prohibitin; POLG, mitochondrial polymerase $\gamma$; POLRMT, mitochondrial RNA polymerase; TFB1M, mitochondrial transcription factor B1; TFB2M, mitochondrial transcription factor $\mathrm{B} 2$.

form of mtDNA renders maintenance of intact nucleoid structure difficult due to hindered spatial organization between the mtDNA and nucleoid-constituting proteins. Eventually, nucleoids encapsulating linearized mtDNA may collapse or be degraded by mitochondrial proteases or nucleases. This was reported following application of mitochondria-targeted obligate heterodimeric zinc finger nucleases capable of specifically eliminating pathogenic human mtDNA, thereby altering the ratio of desired haplotypes [81]. Additionally, the presence of deletion mutations in mtDNA present in human mitochondrial diseases $[12,15]$ suggests that mtDNA size is not a critical factor in nucleoid formation and maintenance; however, additional studies are needed to determine whether the state of mtDNA in either circular or linear form is important in nucleoid construction and maintenance.

Mitochondria are too large and filamentous to achieve spatial uniformity by free diffusion [82]; therefore, mitochondrial nucleoids use active control to ensure their segregation in proportion to the cytoplasmic volume and spacing in semiregular arrays, unlike allocations of chromosomes during cell division [83, 84]. Mitochondrial fission and fusion machinery operate on the two lipid bilayers that surround mitochondria to ensure the efficient distribution of mtDNA throughout the cell and simultaneously protect the nucleoid from fission events at the nucleoid itself [7]. It is likely that the processes of mitochondrial fission, which appears uncoupled to mtDNA replication in nucleoids and occurs adjacent to mtDNA $[7,25]$ and mtDNAnucleoid organization, are coordinated to maintain mitochondrial function [83]. During the fission process, nucleoid-partitioning errors are suppressed by controls at two levels: mitochondrial volume is actively distributed throughout a cell and nucleoids are spaced in semiregular arrays within mitochondria. The fusion process is coordinated by mitofusin proteins and optic atrophy 1 (OPA1), guanosine triphosphatases located at the outer mitochondrial membrane $[9,33]$. OPA1 generates a peptide, including the exon- $4 \mathrm{~b}$ domain associated with the inner membrane and crucial for mtDNA maintenance that directly interacts with nucleoids and allows their distribution within the mitochondrial network to promote mitochondrial genome replication $[85,86]$. It remains unclear what nucleoid-associated factors are intricately connected to the fission and fusion machinery to ensure nucleoid propagation. From an elegant experiment using fluorescent-protein constructs and fusion-protein expression in HepG2 cells, it was speculated that nucleoid redistribution could occur following fission and its subsequent reintegration into the mitochondrial network [65]. However, due to the highly dynamic state of mitochondria and the existing hurdles in visualizing mitochondrial nucleoids in intact cells [65], our understating of nucleoid 
distribution within the mitochondrial network upon network-morphological changes remains limited. Apart from nucleoid redistribution in the cell, it remains unclear whether the nucleoid fission/reintegration process is involved in compensating for or selectively segregating nucleoids containing mutated mtDNA.

\section{6. mtDNA Content in the Mitochondrial Nucleoid}

In mitochondrial genetics, the number of $\operatorname{mtDNA}(\mathrm{s})$ contained within a single nucleoid is a fundamental question that remains somewhat controversial. Strong discrepancies in mtDNA number present in a single nucleoid might be associated with methodological differences, different cell types, or the unveiled complex behaviors of a nucleoid [22, 87]. According to stimulated emission-depletion microscopy or photoactivated light microscopy [22], mammalian cells might contain an average of $1.45 \mathrm{mtDNA}$ molecules per nucleoid (ranging from $\sim 2.4$ to $\sim 7.8$ per nucleoid). However, a recent study of mitochondrial nucleoids from mouse embryonic fibroblasts reported that a single nucleoid could contain more than two mtDNA molecules based on the characteristics of TFAM-mediated mtDNA packaging indicating a spherical shape [87]. These different points regarding mtDNA-molecule population within a single nucleoid highlight the continued importance of understanding nucleoid ultrastructure, but questions concerning control of individual mtDNA transcription and replication nucleoids remain unsolved. Interestingly, mtDNA does not mix between two different nucleoids, despite their proximity in space and time within the mitochondrial network, but rather, mitochondrial nucleoids are tightly regulated by their genetic content rather than the free exchange of mtDNAs [88]. However, heterologous mtDNAs within maximal diffusible distance of mtDNA transcripts in the same mitochondrion can transcomplement to restore mitochondrial function, a result that provides a basis for future research in mitochondrial therapeutics $[32,83]$.

\section{Mitochondrial Nucleoid-Associating Proteins}

In the previous section, the characteristics of mitochondrial nucleoids were briefly addressed (Figures 1-5). Principally, nucleoid-associated proteins can be defined as any protein that either temporarily or permanently associates directly with mtDNA or with other nucleoid proteins and plays roles in mtDNA maintenance [28]. To better understand the biological functions and regulation of mitochondrial nucleoids, identification of proteins involved in nucleoid formation is necessary [89]. Except for conserved TFAM and $\mathrm{mtSSB}[19,53]$, there is no consensus regarding nucleoid composition due to differences in cell types or tissues used for preparations, the various biochemical approaches used for examination based on noncovalent protein-DNA and protein-protein interactions [90], formaldehyde crosslinking [91] or proximity-based biotinylation techniques [43], the low abundance of proteins within mitochondrial nucleoids, and the limited characterization of proteins related to mtDNA maintenance and gene expression [89]. Additionally, it is difficult to use genetic methods to study these associations, because all of the proteins identified are likely required to maintain mitochondrial function [67].

Nucleoids from most organisms contain $\geq 50$ proteins, many of which have not been characterized with respect to nucleoid function $[22,28,66,67,92-94]$. Generally, proteins involved in mtDNA packaging or covering exhibit low molecular weight and function as multimers (Table 1). Nucleoid-associated proteins from various organisms can be classified into at least four groups: (1) proteins with known functions in DNA transactions and packaging, (2) proteins participating in protein quality control, (3) bifunctional metabolic enzymes with various activities, and (4) cytoskeletal components [63]. Some examples of these nucleoidassociated proteins are presented in Table 1 and Figure 5. Among those identified as nucleoid-associated proteins, many exhibit identifiable activities unrelated directly to mtDNA maintenance, suggesting that their bifunctionality might involve participation in both mitochondrial metabolism and mtDNA maintenance $[32,89,95]$. Interestingly, mutations in proteins associated with the mitochondrial nucleoid might cause either the loss of mtDNA content from the cell or generation of mtDNA mutations $[32,96]$.

7.1. Mitochondrial TFAM. TFAM is a nuclear-DNA-encoded $24-\mathrm{kDa}$ protein containing two high-mobility group(HMG-) box domains and able to bend, wrap, and unwind DNA through modes involving sliding, collisions, and patch formation $[29,89,90,97-101]$. TFAMs cover mtDNA with a footprint of between $10 \mathrm{bp}$ and $30 \mathrm{bp}$ (Figure 3) and mediate the tight compaction of relaxed mtDNA [62]. In addition to a role as a master transcription factor, TFAM plays an equally important role in promoter selection, initiation of genome replication, and the regulation of mtDNA copy number [98]. TFAM concentration can increase mtDNA content through its preferential binding at the light-strand promoter (LSP) in the D-loop and TFAMmediated stabilization of mtDNA, perhaps by reducing the rate of DNA turnover [98]. The molecular ratio of TFAM relative to mtDNA is 900:1 in human mitochondria [102], with this amount of TFAM sufficient to coat $16.6 \mathrm{kbp}$ circular human mtDNA [96]. Theoretically, mtDNA density, TFAM/ mtDNA stoichiometry, or TFAM density within a single nucleoid may differ under various physiological (mtDNA transcription or replication) or pathological conditions [64], thereby implying the presence of mechanisms that select only a subset of mtDNA molecules for replication, with others remaining in a silent state [101]. A recent finding suggested that human TFAM plays an important role in the equal distribution and symmetric segregation of mtDNA in cultured cells [66]. For example, enlarged mtDNA nucleoids have been observed in both TFAM-knockdown HeLa cells and TFAM-overexpressing mice [103]. The process of uncoating mtDNA has not been elucidated but likely involves the selective and processive dissociation of TFAM [104]. In view of TFAM turnover [7], LONP1 determines the proteolytic degradation of TFAM and constitutes an additional step in controlling mtDNA content (Figure 3). Apart from regulating 


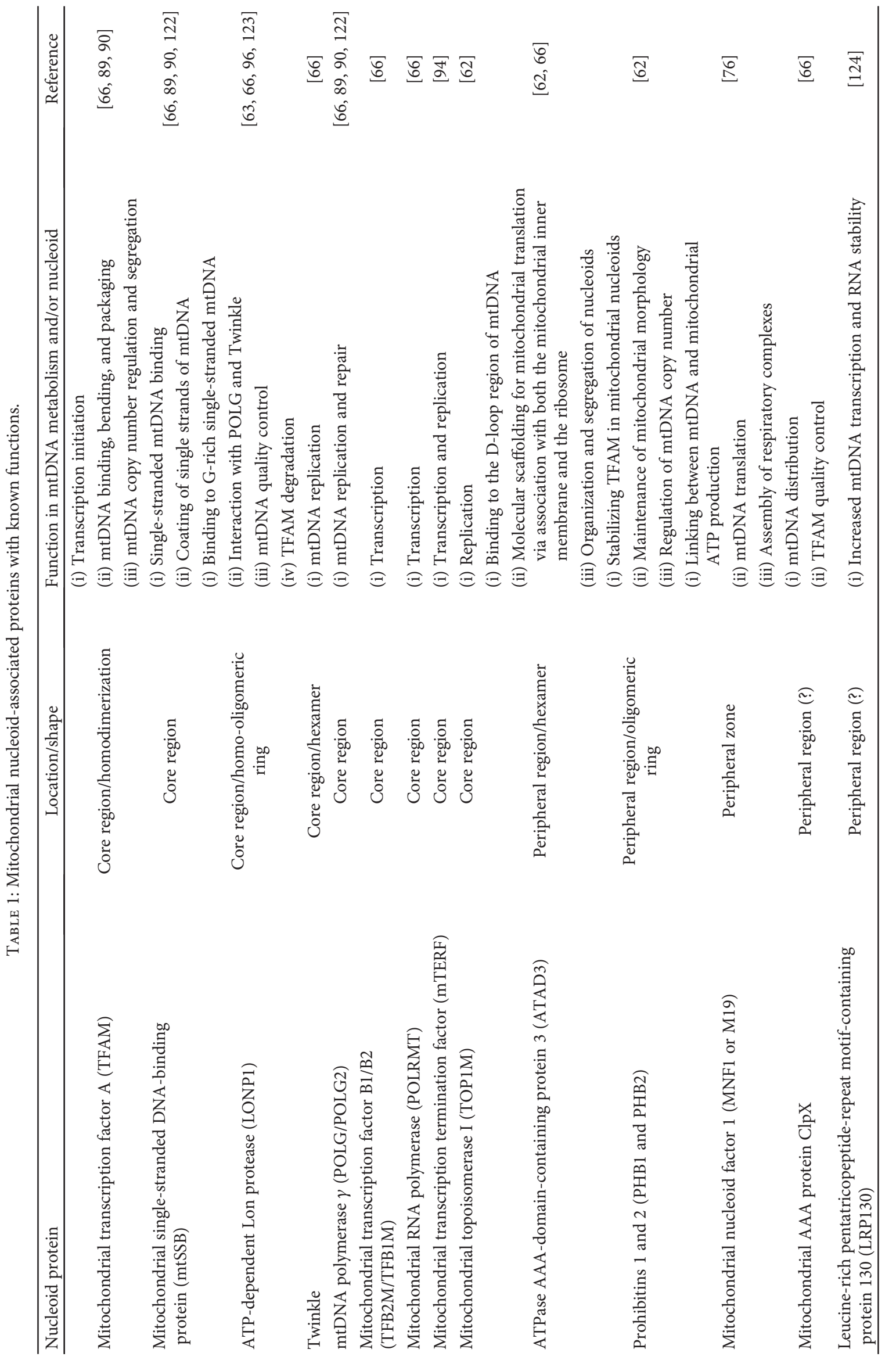


TFAM expression and turnover, posttranslation modification of TFAM by glycosylation, phosphorylation, acetylation, or ubiquitination might constitute alternative control points of TFAM activity, given that these modifications can influence DNA-binding activity, protein-protein interactions, homodimerization, or cooperative-binding characteristics [98]. For example, TFAM can be phosphorylated within its HMG1 domain by cyclic adenosine monophosphate-dependent protein kinase in mitochondria, thereby impairing its ability to bind DNA and activate transcription [104]. By contrast, in the cytosol, TFAM phosphorylation might alter its degradation by the proteasome or its association with mitochondrial protein-translocation machinery [104].

7.2. Mitochondrial Transcription and Replication Machinery. The minimal proteins required for mtDNA transcription (e.g., POLRMT and mitochondrial transcription factor $B$ (mtTFB)) and replication (POLG and POLG2) are embedded in the core region of nucleoids through their mtDNAbinding capabilities $[13,43,62]$. Due to the high degree of compaction in mtDNA, mitochondrial topoisomerase 1, found in the core region of mitochondrial nucleoids, is required during replication to ease torsional strain resulting from replication progression [62]. Additionally, POLRMT and $\mathrm{mtTFB}$, located in the core region of nucleoids, are necessary for mitochondrial transcription $[13,62]$.

7.3. $m t S S B$. $\mathrm{mtSSB}$ is a $16-\mathrm{kDa}$ protein that forms a tetramer and binds ssDNA with high affinity in a sequenceindependent manner, thereby aiding DNA replication, recombination, and repair processes $[64,105]$. Similar to TFAM, mtSSB is a major nucleoid-associated protein also involved in mtDNA/nucleoid distribution within the mitochondrial network [106]. Additionally, mtSSB influences mitochondrial biogenesis $[105,106]$, and its downregulation leads to increases in morphological alterations, such as fragmentation or elongation, of mitochondria [107].

7.4. Twinkle Helicase. Twinkle helicase is a nucleoidassociated protein found in the core region [66] and the only known mitochondrial helicase involved in unwinding mtDNA during the replication process, synthesis of the nascent D-loop strand, and completion of mtDNA replication [108, 109]. Decreases in Twinkle helicase concentration result in mtDNA depletion, whereas overexpression leads to increases in mtDNA copy number [109]. Many disease-causing mutations, including autosomal dominant progressive external opthalmoplegia, have been mapped to the Twinkle helicase gene, with mutation resulting in defects in OXPHOS and the onset of neuromuscular symptoms [109]. Twinkle helicase might also promote nucleoid attachment to membrane structures highly enriched in cholesterol, thereby providing a replication platform at ER-mitochondrial junctions [71].

7.5. Mitochondrial ATPase AAA-Domain-Containing Proteins (AAA). Several ATP-dependent proteases, including LONP1, ATP-dependent Clp protease ATP-binding subunit ClpX-like protein, and m-AAA protease, localize to the mitochondrial matrix [96] and are associated with the peripheral region of nucleoids $[61,96,110]$. Among these, LONP1 is a quality control enzyme that degrades oxidatively modified and misfolded proteins and also binds to specific regions of the mitochondrial genome, including ssDNA in both the LSP region and RNA produced from the LSP region [98]. Additionally, LONP1 might recognize oxidized TFAM or degrade unbound TFAM; alternatively, LONP1 can also remove TFAM from oxidatively modified DNA, to which TFAM binds with higher efficiency than it does unmodified DNA [98]. Although the triggers for LONP1-mediated TFAM degradation remain unclear, mitochondrial stress might activate LONP1 activity to initiate TFAM degradation and activate transcription in quiescent mtDNAs [98]. Interestingly, LONP1 expression decreases with age or exposure to chronic stress, possibly resulting in accumulation of oxidized proteins and disturbance of the nucleoid dynamics [111].

7.6. ATAD3. ATAD3A and the less abundant ATAD3B are protein paralogs that form heterohexamers or homohexamers with ATAD3A and extend from the inner membrane into the outer mitochondrial membrane $[66,112]$. ATAD3 was discovered as an important membrane-bound mitochondrial ATPase [112]. Although ATAD3 appeared to be bound to the D-loop of mtDNA in nucleoid [75], subsequent experiments indicated that ATAD3 made direct contact with mtDNA but is among the nucleoid-associated proteins involved in connections between mitochondrial nucleoids and mitochondrial ribosomes $[66,93]$. ATAD3 associates with ER-mitochondrial junctions and holds together Twinkle helicase-containing mammalian nucleoids attached to membrane structures highly enriched in cholesterol [71]. Therefore, ATAD3 also plays an important role in nucleoid positioning in human mitochondria, with altered ATAD3 expression disturbing mtDNA maintenance and replication [77].

7.7. Prohibitin. Prohibitin proteins (PHB1 and PHB2) are membrane-anchored molecular chaperones and protein stabilizers [67, 103]. In addition to pleiotropic functions, including apoptosis, in mitochondria, $\mathrm{PHB} 1$ is required for the organization and stability of mitochondrial nucleoids either through a TFAM-dependent or through a TFAMindependent pathway, in which it regulates nucleoid organization directly or through undefined nucleoid factors [113]. Several reports supported the notion that PHBs are important in mtDNA copy number regulation $[103,113]$.

7.8. Other Putative Nucleoid-Associated Proteins. A group of heat-shock proteins (HSPs) are associated with nucleoids in both yeast and human cells [63]. HSP60 functions both in mitochondrial protein import and as a nucleoid protein required for nucleoid division [63]. The components of detergent-resistant mtDNA nucleoids include adenine nucleotide translocator (ANT), the E2 subunits of two large dehydrogenase complexes, pyruvate dehydrogenase, and branched-chain keto acid dehydrogenase without association with other subunits [67]. 


\section{Links between Mitochondrial Nucleoid Composition and Metabolic Control}

Mitochondrial nucleoids undergo remodeling, such as transition of its structure or recruitment of other proteins that influence nucleoid-related activities in response to metabolic cues in yeast $[114,115]$. There is less concrete evidence of yeast-like nucleoid remodeling in mammalian systems; however, nucleoid remodeling might be possible according to metabolic demand $[5,32]$, because access to mtDNA for transcription, translation, and replication is highly coordinated by various factors inside and outside of the nucleoid compartment. Moreover, nucleoid-associated proteins are directly involved in not only mtDNA maintenance and propagation but also metabolic activities not directly linked to mtDNA stability [62]. Additionally, retrograde signaling from the mitochondria to the nucleus can be promoted through interactions between mtDNA and nucleoidassociated factors $[2,7]$. It is unclear whether nucleoidassociated proteins can directly regulate mitochondrial gene expression or bioenergetics.

\section{Pathological Changes Associated with Mitochondrial Nucleoids}

Mitochondrial morphology is coupled to function, as a loss in mitochondrial bioenergetic capacity results in an inability to maintain a highly ordered structure [62]. The shaping, maintenance, and dissociation of nucleoids in a mitochondrion is undertaken by numerous proteins that communicate with one another and the nucleoid in order to determine cellular demands dependent upon physiological conditions. The general principles of nucleoid organization and its pathological implications remain unclear; however, significant interest in the role of nucleoids and their impact on mitochondriarelated diseases has focused on their association with the accumulation of numerous mtDNA mutations [27]. Mutations in mtDNA and/or aberrant nucleoid organization might be a causal factor in etiologies of various diseases, including cancers $[15,106]$. In addition to mutations or damage to nucleoid-associated proteins, aberrant interactions between or dysfunction of nucleoid-interacting proteins causes pathological conditions due to failed mtDNA maintenance. For example, ANT1 interacts with mtDNA [67], and its mutation causes a genetic disorder leading to multiple mtDNA deletions and autosomal dominant progressive external opthalmoplegia [116]. Additionally, the subunits of complex I and the E2 subunits of ATP synthase and 2-oxo-acid dehydrogenase have been identified in nucleoids and are involved in mitochondrial diseases and aging [67]. Under physiological or various cellular stress conditions, p53 can maintain nuclear genome stability through the repair of damaged DNA and the integration of cell-death-signaling pathways with DNA-damage checkpoints [117]. Recently, an additional role for p53 as guardian of the mitochondrial genome was suggested [118]. Mitochondria-translocated p53 can interact with TFAM and POLG located in the core region of nucleoids and involved in mtDNA maintenance [119]. It was suggested that the expression of dynamin-related protein 1 and OPA1 involved in mitochondrial dynamics is regulated by p53 [118]. Interestingly, human mtDNA also contains a putative p53-binding sequence [120], suggesting that p53 functions involve both the nuclear and mitochondrial subcellular compartments and are responsible for maintaining mtDNA integrity through its activities in both regions (Figure 5). It remains unclear whether p53 directly affects the structure and dynamics of mitochondrial nucleoids.

Oxidative damage can disturb the regulation of nucleoid dynamics. For example, oxidized mtDNA is degraded by lysosomes; however, oxidized mitochondrial nucleoids are not degraded via the lysosomal pathway in neutrophils in human lupus, resulting in activation of type I interferon production [121]. Oxidative stress may deteriorate the dynamics of nucleoids due to their resulting structural modifications and the breakdown of redox control, resulting in mitochondrial dysfunction. However, more extensive work is needed to clarify the mechanisms associated with oxidative-stress-mediated disruption and/or dysfunction of mitochondrial nucleoids. The clustering of multiple mtDNA genomes into a single nucleoid complex might promote the progressive age-related accumulation of deletions and point mutations in mtDNA in many somatic tissues and particularly in postmitotic cells. By contrast, oocytes appear to have the ability to select against deleterious mutations in mtDNA, at least in mice [17]. Therefore, the processes by which nucleoids are actively chosen for mtDNA replication and distribution within mitochondrial networks are not clearly understood and remain as highly relevant issues associated with understanding the basis of human metabolic diseases, aging, and neurodegenerative disorders caused by mtDNA mutations, as well as those in nuclear genes, that affect mtDNA maintenance [70].

\section{Concluding Remarks and Perspectives}

In mitochondrial biology and its role in human diseases, nucleoids remain an unexplored feature. Their role as entities that organize mtDNA by forming complexes with accessory proteins, as well as regulators of gene expression, greatly influences the phenotypic expression of mtDNA defects [17]. In this review, we addressed the newly emerging field of nucleoid research, including investigations of its structure and dynamic regulation. Nucleoid-associated proteins function as building blocks of nucleoids, which are intimately involved in mitochondrial genetics and the fine tuning of metabolic demands (Figure 5). To understand the complex behavior of nucleoids, it will be necessary to examine the specific interactions between different nucleoid-associated proteins and mtDNA to definitively elucidate their roles in nucleoid organization. In addition to posttranslational modifications of nucleoid-associated proteins, oxidative changes that occur in nucleoid-associated proteins and their impact on mtDNA likely influence nucleoid dynamics and function and might be necessary to understand the real functional role of nucleoid and mitochondria. Furthermore, the assembly and dynamic control of nucleoid 
structure involving mtDNA also remains unclear and should be the subject of future investigation.

Collectively, mtDNA is preserved in a highly ordered manner by nucleoids. Mitochondrial nucleoids act not as simple shields or parcels for mtDNA but constitute a switch for controlling mitochondrial metabolism in response to cellular demands. New findings associated with mitochondria should be interpreted in conjunction with nucleoid dynamics to fully understand its overall physiological and pathophysiological role.

\section{Conflicts of Interest}

The authors declare that there are no conflicts of interest.

\section{Acknowledgments}

The authors apologize for the vast number of outstanding publications that could not be cited due to space limitations. This work was supported by the Priority Research Centers Program (2010-0020224) and the Basic Science Research Program (2015R1A2A1A13001900 and 2015R1D1A3A 01015596) through the National Research Foundation of Korea (NRF) funded by the Ministry of Education, Science, and Technology.

\section{References}

[1] A. Kaniak-Golik and A. Skoneczna, "Mitochondria-nucleus network for genome stability," Free Radical Biology \& Medicine, vol. 82, pp. 73-104, 2015.

[2] F. M. da Cunha, N. Q. Torelli, and A. J. Kowaltowski, "Mitochondrial retrograde signaling: triggers, pathways, and outcomes," Oxidative Medicine and Cellular Longevity, vol. 2015, Article ID 482582, 10 pages, 2015.

[3] M. Guha and N. G. Avadhani, "Mitochondrial retrograde signaling at the crossroads of tumor bioenergetics, genetics and epigenetics," Mitochondrion, vol. 13, no. 6, pp. 577591, 2013.

[4] H. M. McBride, M. Neuspiel, and S. Wasiak, "Mitochondria: more than just a powerhouse," Current Biology, vol. 16, no. 14, pp. R551-RR60, 2006.

[5] I. Bohovych and O. Khalimonchuk, "Sending out an SOS: mitochondria as a signaling hub," Frontiers in Cell and Development Biology, vol. 4, no. 109, pp. 1-15, 2016.

[6] I. E. Scheffler, Mitochondria, John Wiley \& Sons, Inc., New Jersey, 2nd edition, 2008.

[7] F. J. Iborra, H. Kimura, and P. R. Cook, "The functional organization of mitochondrial genomes in human cells," $B M C$ Biology, vol. 2, no. 1, p. 9, 2004.

[8] N. Schwarz and R. E. Leube, "Intermediate filaments as organizers of cellular space: how they affect mitochondrial structure and function," Cell, vol. 5, no. 3, p. 30, 2016.

[9] S. Campello and L. Scorrano, "Mitochondrial shape changes: orchestrating cell pathophysiology," EMBO Reports, vol. 11, no. 9, pp. 678-684, 2010.

[10] I. E. Scheffler, "Biogenesis of mitochondria," in Mitochondria, pp. 60-167, John Wiley \& Sons, Inc., Hoboken, NJ, USA, 2007.
[11] M. Schwartz and J. Vissing, "Paternal inheritance of mitochondrial DNA," The New England Journal of Medicine, vol. 347, no. 8, pp. 576-580, 2002.

[12] R. W. Taylor and D. M. Turnbull, "Mitochondrial DNA mutations in human disease," Nature Reviews. Genetics, vol. 6, no. 5, pp. 389-402, 2005.

[13] M. Falkenberg, N.-G. Larsson, and C. M. Gustafsson, "DNA replication and transcription in mammalian mitochondria," Annual Review of Biochemistry, vol. 76, pp. 679-699, 2007.

[14] B. Thornton, B. Cohen, W. Copeland, and B. L. Maria, "Mitochondrial disease: clinical aspects, molecular mechanisms, translational science, and clinical frontiers," Journal of Child Neurology, vol. 29, no. 9, pp. 1179-1207, 2014.

[15] S. Lee, N. Kim, Y. Noh et al., "Mitochondrial DNA, mitochondrial dysfunction, and cardiac manifestations," Frontiers in Bioscience (Landmark Edition), vol. 21, pp. 1410-1426, 2016.

[16] H. Huang and K. G. Manton, "The role of oxidative damage in mitochondria during aging: a review," Frontiers in Bioscience, vol. 9, pp. 1100-1117, 2004.

[17] D. F. Bogenhagen, "Does mtDNA nucleoid organization impact aging?” Experimental Gerontology, vol. 45, no. 7-8, pp. 473-477, 2010.

[18] C. B. Park and N. G. Larsson, "Mitochondrial DNA mutations in disease and aging," The Journal of Cell Biology, vol. 193, no. 5, pp. 809-818, 2011.

[19] Y. Wang and D. F. Bogenhagen, "Human mitochondrial DNA nucleoids are linked to protein folding machinery and metabolic enzymes at the mitochondrial inner membrane," Journal of Biological Chemistry, vol. 281, no. 35, pp. 2579125802, 2006.

[20] F. Legros, F. Malka, P. Frachon, A. Lombes, and M. Rojo, "Organization and dynamics of human mitochondrial DNA," Journal of Cell Science, vol. 117, no. Part 13, pp. 2653-2662, 2004.

[21] J. Prachar, "Ultrastructure of mitochondrial nucleoid and its surroundings," General Physiology and Biophysics, vol. 35, no. 3, pp. 273-286, 2016.

[22] D. F. Bogenhagen, "Mitochondrial DNA nucleoid structure," Biochimica et Biophysica Acta, vol. 1819, no. 9-10, pp. 914920, 2012.

[23] D. Bogenhagen and D. A. Clayton, "The number of mitochondrial deoxyribonucleic acid genomes in mouse $\mathrm{L}$ and human HeLa cells. Quantitative isolation of mitochondrial deoxyribonucleic acid," The Journal of Biological Chemistry, vol. 249, no. 24, pp. 7991-7995, 1974.

[24] R. Garesse and C. G. Vallejo, "Animal mitochondrial biogenesis and function: a regulatory cross-talk between two genomes," Gene, vol. 263, no. 1-2, pp. 1-16, 2001.

[25] R. Ban-Ishihara, T. Ishihara, N. Sasaki, K. Mihara, and N. Ishihara, "Dynamics of nucleoid structure regulated by mitochondrial fission contributes to cristae reformation and release of cytochrome c," Proceedings of the National Academy of Sciences of the United States of America, vol. 110, no. 29, pp. 11863-11868, 2013.

[26] G. L. Ciesielski, M. Plotka, M. Manicki et al., "Nucleoid localization of Hsp40 Mdj1 is important for its function in maintenance of mitochondrial DNA," Biochimica et Biophysica Acta, vol. 1833, no. 10, pp. 2233-2243, 2013.

[27] A. A. Kolesnikov, "The mitochondrial genome. The nucleoid," Biochemistry (Moscow), vol. 81, no. 10, pp. 1057-1065, 2016. 
[28] J. N. Spelbrink, "Functional organization of mammalian mitochondrial DNA in nucleoids: history, recent developments, and future challenges," IUBMB Life, vol. 62, no. 1, pp. 19-32, 2010.

[29] I. J. Holt, J. He, C. C. Mao et al., "Mammalian mitochondrial nucleoids: organizing an independently minded genome," Mitochondrion, vol. 7, no. 5, pp. 311-321, 2007.

[30] S. Meeusen and J. Nunnari, "Evidence for a two membrane-spanning autonomous mitochondrial DNA replisome," The Journal of Cell Biology, vol. 163, no. 3, pp. 503-510, 2003.

[31] M. Navratil, B. G. Poe, and E. A. Arriaga, "Quantitation of DNA copy number in individual mitochondrial particles by capillary electrophoresis," Analytical Chemistry, vol. 79, no. 20, pp. 7691-7699, 2007.

[32] R. W. Gilkerson, "Mitochondrial DNA nucleoids determine mitochondrial genetics and dysfunction," The International Journal of Biochemistry \& Cell Biology, vol. 41, no. 10, pp. 1899-1906, 2009.

[33] J. G. McCarron, C. Wilson, M. E. Sandison et al., "From structure to function: mitochondrial morphology, motion and shaping in vascular smooth muscle," Journal of Vascular Research, vol. 50, no. 5, pp. 357-371, 2013.

[34] P. Jezek and L. Plecita-Hlavata, "Mitochondrial reticulum network dynamics in relation to oxidative stress, redox regulation, and hypoxia," The International Journal of Biochemistry \& Cell Biology, vol. 41, no. 10, pp. 1790-1804, 2009.

[35] A. Dlaskova, H. Engstova, L. Plecita-Hlavata et al., "Distribution of mitochondrial DNA nucleoids inside the linear tubules vs. bulk parts of mitochondrial network as visualized by $4 \mathrm{Pi}$ microscopy," Journal of Bioenergetics and Biomembranes, vol. 47, no. 3, pp. 255-263, 2015.

[36] G. Twig, A. Elorza, A. J. Molina et al., "Fission and selective fusion govern mitochondrial segregation and elimination by autophagy," The EMBO Journal, vol. 27, no. 2, pp. 433-446, 2008.

[37] W. Kuhlbrandt, "Structure and function of mitochondrial membrane protein complexes," BMC Biology, vol. 13, no. 1, p. $89,2015$.

[38] J. Bereiter-Hahn, M. Voth, S. Mai, and M. Jendrach, "Structural implications of mitochondrial dynamics," Biotechnology Journal, vol. 3, no. 6, pp. 765-780, 2008.

[39] A. E. Frazier, C. Kiu, D. Stojanovski, N. J. Hoogenraad, and M. T. Ryan, "Mitochondrial morphology and distribution in mammalian cells," Biological Chemistry, vol. 387, no. 12, pp. 1551-1558, 2006.

[40] C. Vasquez-Trincado, I. Garcia-Carvajal, C. Pennanen et al., "Mitochondrial dynamics, mitophagy and cardiovascular disease," The Journal of Physiology, vol. 594, no. 3, pp. 509525, 2015.

[41] H. Schagger and K. Pfeiffer, "Supercomplexes in the respiratory chains of yeast and mammalian mitochondria," The EMBO Journal, vol. 19, no. 8, pp. 1777-1783, 2000.

[42] B. Kornmann and P. Walter, "ERMES-mediated ERmitochondria contacts: molecular hubs for the regulation of mitochondrial biology," Journal of Cell Science, vol. 123, Part 9, pp. 1389-1393, 2010.

[43] S. U. Liyanage, E. Coyaud, E. M. Laurent et al., "Characterizing the mitochondrial DNA polymerase gamma interactome by BioID identifies Ruvbl2 localizes to the mitochondria," Mitochondrion, vol. 32, pp. 31-35, 2016.
[44] M. Harner, C. Körner, D. Walther et al., "The mitochondrial contact site complex, a determinant of mitochondrial architecture," The EMBO Journal, vol. 30, no. 21, pp. 4356-4370, 2011.

[45] K. Kehrein, R. Schilling, B. V. Möller-Hergt et al., "Organization of mitochondrial gene expression in two distinct ribosome-containing assemblies," Cell Reports, vol. 10, no. 6, pp. 843-853, 2015.

[46] A. Chacinska, P. Rehling, B. Guiard et al., "Mitochondrial translocation contact sites: separation of dynamic and stabilizing elements in formation of a TOM-TIM-preprotein supercomplex," The EMBO Journal, vol. 22, no. 20, pp. 5370-5381, 2003.

[47] M. M. Nass and S. Nass, "Intramitochondrial fibers with DNA characteristics. I. Fixation and electron staining reactions," The Journal of Cell Biology, vol. 19, pp. 593-611, 1963.

[48] C. Meisinger, A. Sickmann, and N. Pfanner, "The mitochondrial proteome: from inventory to function," Cell, vol. 134, no. 1, pp. 22-24, 2008.

[49] N. D. Bonawitz, D. A. Clayton, and G. S. Shadel, "Initiation and beyond: multiple functions of the human mitochondrial transcription machinery," Molecular Cell, vol. 24, no. 6, pp. 813-825, 2006.

[50] M. G. Claros, J. Perea, Y. Shu, F. A. Samatey, J. L. Popot, and C. Jacq, "Limitations to in vivo import of hydrophobic proteins into yeast mitochondria. The case of a cytoplasmically synthesized apocytochrome b," European Journal of Biochemistry, vol. 228, no. 3, pp. 762-771, 1995.

[51] S. Anderson, A. T. Bankier, B. G. Barrell et al., "Sequence and organization of the human mitochondrial genome," Nature, vol. 290, no. 5806, pp. 457-465, 1981.

[52] J. Magnusson, M. Orth, P. Lestienne, and J. W. Taanman, "Replication of mitochondrial DNA occurs throughout the mitochondria of cultured human cells," Experimental Cell Research, vol. 289, no. 1, pp. 133-142, 2003.

[53] D. Kang and N. Hamasaki, "Mitochondrial transcription factor A in the maintenance of mitochondrial DNA: overview of its multiple roles," Annals of the New York Academy of Sciences, vol. 1042, no. 1, pp. 101-108, 2005.

[54] N. Suematsu, H. Tsutsui, J. Wen et al., "Oxidative stress mediates tumor necrosis factor-alpha-induced mitochondrial DNA damage and dysfunction in cardiac myocytes," Circulation, vol. 107, no. 10, pp. 1418-1423, 2003.

[55] D. C. Wallace, "Mitochondrial diseases in man and mouse," Science, vol. 283, no. 5407, pp. 1482-1488, 1999.

[56] J. B. Stewart and P. F. Chinnery, "The dynamics of mitochondrial DNA heteroplasmy: implications for human health and disease," Nature Reviews. Genetics, vol. 16, no. 9, pp. 530-542, 2015.

[57] D. M. Krzywanski, D. R. Moellering, J. L. Fetterman, K. J. Dunham-Snary, M. J. Sammy, and S. W. Ballinger, "The mitochondrial paradigm for cardiovascular disease susceptibility and cellular function: a complementary concept to Mendelian genetics," Laboratory Investigation, vol. 91, no. 8, pp. 1122-1135, 2011.

[58] J. Wang, F. Lin, L. L. Guo, X. J. Xiong, and X. Fan, "Cardiovascular disease, mitochondria, and traditional Chinese medicine," Evidence-Based Complementary and Alternative Medicine, vol. 2015, Article ID 143145, 7 pages, 2015.

[59] M. Scheibye-Knudsen, E. F. Fang, D. L. Croteau, D. M. Wilson Iii, and V. A. Bohr, "Protecting the mitochondrial 
powerhouse," Trends in Cell Biology, vol. 25, no. 3, pp. 158-170, 2015.

[60] C. Robinow and E. Kellenberger, "The bacterial nucleoid revisited," Microbiological Reviews, vol. 58, no. 2, pp. 211232, 1994.

[61] L. Ambro, V. Pevala, J. Bauer, and E. Kutejova, "The influence of ATP-dependent proteases on a variety of nucleoidassociated processes," Journal of Structural Biology, vol. 179, no. 2, pp. 181-192, 2012.

[62] R. Gilkerson, L. Bravo, I. Garcia et al., "The mitochondrial nucleoid: integrating mitochondrial DNA into cellular homeostasis," Cold Spring Harbor Perspectives in Biology, vol. 5, no. 5, article a011080, 2013.

[63] M. Kucej and R. A. Butow, "Evolutionary tinkering with mitochondrial nucleoids," Trends in Cell Biology, vol. 17, no. 12, pp. 586-592, 2007.

[64] L. Alan, T. Spacek, and P. Jezek, "Delaunay algorithm and principal component analysis for $3 \mathrm{D}$ visualization of mitochondrial DNA nucleoids by Biplane FPALM/dSTORM," European Biophysics Journal, vol. 45, no. 5, pp. 443-461, 2016.

[65] J. Tauber, A. Dlaskova, J. Santorova et al., "Distribution of mitochondrial nucleoids upon mitochondrial network fragmentation and network reintegration in HEPG2 cells," The International Journal of Biochemistry \& Cell Biology, vol. 45, no. 3, pp. 593-603, 2013.

[66] D. F. Bogenhagen, D. Rousseau, and S. Burke, "The layered structure of human mitochondrial DNA nucleoids," The Journal of Biological Chemistry, vol. 283, no. 6, pp. 36653675,2008

[67] D. F. Bogenhagen, Y. Wang, E. L. Shen, and R. Kobayashi, "Protein components of mitochondrial DNA nucleoids in higher eukaryotes," Molecular \& Cellular Proteomics, vol. 2, no. 11, pp. 1205-1216, 2003.

[68] C. Kukat and N.-G. Larsson, "mtDNA makes a U-turn for the mitochondrial nucleoid," Trends in Cell Biology, vol. 23, no. 9, pp. 457-463, 2013.

[69] H. Li, Y. Ruan, K. Zhang et al., "Mic60/Mitofilin determines MICOS assembly essential for mitochondrial dynamics and mtDNA nucleoid organization," Cell Death and Differentiation, vol. 23, no. 3, pp. 380-392, 2016.

[70] S. C. Lewis, L. F. Uchiyama, and J. Nunnari, "ER-mitochondria contacts couple mtDNA synthesis with mitochondrial division in human cells," Science, vol. 353, no. 6296, p. aaf5549, 2016.

[71] J. M. Gerhold, S. Cansiz-Arda, M. Lohmus et al., "Human mitochondrial DNA-protein complexes attach to a cholesterol-rich membrane structure," Scientific Reports, vol. 5, no. 15292, pp. 1-14, 2015.

[72] V. M. Pastukh, O. M. Gorodnya, M. N. Gillespie, and M. V. Ruchko, "Regulation of mitochondrial genome replication by hypoxia: the role of DNA oxidation in D-loop region," Free Radical Biology \& Medicine, vol. 96, pp. 78-88, 2016.

[73] X. Z. Chen, Y. Fang, Y. H. Shi et al., "Deciphering the spectrum of somatic mutations in the entire mitochondrial DNA genome," Genetics and Molecular Research, vol. 14, no. 2, pp. 4331-4337, 2015.

[74] M. Di Re, H. Sembongi, J. He et al., "The accessory subunit of mitochondrial DNA polymerase gamma determines the DNA content of mitochondrial nucleoids in human cultured cells," Nucleic Acids Research, vol. 37, no. 17, pp. 5701-5713, 2009.

[75] J. He, C. C. Mao, A. Reyes et al., "The AAA+ protein ATAD3 has displacement loop binding properties and is involved in mitochondrial nucleoid organization," The Journal of Cell Biology, vol. 176, no. 2, pp. 141-146, 2007.

[76] M. Sumitani, K. Kasashima, E. Ohta, D. Kang, and H. Endo, "Association of a novel mitochondrial protein M19 with mitochondrial nucleoids," Journal of Biochemistry, vol. 146, no. 5, pp. 725-732, 2009.

[77] A. Hubstenberger, N. Merle, R. Charton, G. Brandolin, and D. Rousseau, "Topological analysis of ATAD3A insertion in purified human mitochondria," Journal of Bioenergetics and Biomembranes, vol. 42, no. 2, pp. 143-150, 2010.

[78] L. Alan, T. Spacek, D. Pajuelo Reguera, M. Jaburek, and P. Jezek, "Mitochondrial nucleoid clusters protect newly synthesized mtDNA during doxorubicin- and ethidium bromideinduced mitochondrial stress," Toxicology and Applied Pharmacology, vol. 302, pp. 31-40, 2016.

[79] R. W. Gilkerson, D. H. Margineantu, R. A. Capaldi, and J. M. Selker, "Mitochondrial DNA depletion causes morphological changes in the mitochondrial reticulum of cultured human cells," FEBS Letters, vol. 474, no. 1, pp. 1-4, 2000.

[80] N. Garrido, L. Griparic, E. Jokitalo, J. Wartiovaara, A. M. van der Bliek, and J. N. Spelbrink, "Composition and dynamics of human mitochondrial nucleoids," Molecular Biology of the Cell, vol. 14, no. 4, pp. 1583-1596, 2003.

[81] P. A. Gammage, J. Rorbach, A. I. Vincent, E. J. Rebar, and M. Minczuk, "Mitochondrially targeted ZFNs for selective degradation of pathogenic mitochondrial genomes bearing large-scale deletions or point mutations," EMBO Molecular Medicine, vol. 6, no. 4, pp. 458-466, 2014.

[82] R. Jajoo, Y. Jung, D. Huh et al., "Accurate concentration control of mitochondria and nucleoids," Science, vol. 351, no. 6269 , pp. 169-172, 2016.

[83] E. A. Schon and R. W. Gilkerson, "Functional complementation of mitochondrial DNAs: mobilizing mitochondrial genetics against dysfunction," Biochimica et Biophysica Acta, vol. 1800, no. 3, pp. 245-249, 2010.

[84] Y. H. Chan and W. F. Marshall, "How cells know the size of their organelles," Science, vol. 337, no. 6099, pp. 1186-1189, 2012.

[85] G. Elachouri, S. Vidoni, C. Zanna et al., "OPA1 links human mitochondrial genome maintenance to mtDNA replication and distribution," Genome Research, vol. 21, no. 1, pp. 1220, 2011.

[86] T. Landes, I. Leroy, A. Bertholet et al., "OPA1 (dys)functions," Seminars in Cell \& Developmental Biology, vol. 21, no. 6, pp. 593-598, 2010.

[87] C. Kukat, K. M. Davies, C. A. Wurm et al., "Cross-strand binding of TFAM to a single mtDNA molecule forms the mitochondrial nucleoid," Proceedings of the National Academy of Sciences of the United States of America, vol. 112, no. 36, pp. 11288-11293, 2015.

[88] R. W. Gilkerson, E. A. Schon, E. Hernandez, and M. M. Davidson, "Mitochondrial nucleoids maintain genetic autonomy but allow for functional complementation," The Journal of Cell Biology, vol. 181, no. 7, pp. 1117-1128, 2008.

[89] F. Hensen, S. Cansiz, J. M. Gerhold, and J. N. Spelbrink, "To be or not to be a nucleoid protein: a comparison of massspectrometry based approaches in the identification of 
potential mtDNA-nucleoid associated proteins," Biochimie, vol. 100, pp. 219-226, 2014.

[90] N. Rajala, F. Hensen, H. J. Wessels, D. Ives, J. Gloerich, and J. N. Spelbrink, "Whole cell formaldehyde cross-linking simplifies purification of mitochondrial nucleoids and associated proteins involved in mitochondrial gene expression," PLoS One, vol. 10, no. 2, article e0116726, 2015.

[91] D. F. Bogenhagen, "Biochemical isolation of mtDNA nucleoids from animal cells," Methods in Molecular Biology, vol. 554, pp. 3-14, 2009.

[92] K. Kasashima, M. Sumitani, and H. Endo, "Maintenance of mitochondrial genome distribution by mitochondrial AAA + protein ClpX," Experimental Cell Research, vol. 318, no. 18 , pp. 2335-2343, 2012.

[93] J. He, H. M. Cooper, A. Reyes et al., "Mitochondrial nucleoid interacting proteins support mitochondrial protein synthesis," Nucleic Acids Research, vol. 40, no. 13, pp. 6109-6121, 2012.

[94] A. P. Rebelo, L. M. Dillon, and C. T. Moraes, "Mitochondrial DNA transcription regulation and nucleoid organization," Journal of Inherited Metabolic Disease, vol. 34, no. 4, pp. 941-951, 2011.

[95] M. Macierzanka, M. Plotka, D. Pryputniewicz-Drobinska, A. Lewandowska, R. Lightowlers, and J. Marszalek, "Maintenance and stabilization of mtDNA can be facilitated by the DNA-binding activity of Ilv5p," Biochimica et Biophysica Acta, vol. 1783, no. 1, pp. 107-117, 2008.

[96] Y. Matsushima and L. S. Kaguni, "Matrix proteases in mitochondrial DNA function," Biochimica et Biophysica Acta, vol. 1819, no. 9-10, pp. 1080-1087, 2012.

[97] G. Farge, N. Laurens, O. D. Broekmans et al., "Protein sliding and DNA denaturation are essential for DNA organization by human mitochondrial transcription factor A," Nature Communications, vol. 3, article 1013, pp. 1-9, 2012.

[98] C. T. Campbell, J. E. Kolesar, and B. A. Kaufman, "Mitochondrial transcription factor A regulates mitochondrial transcription initiation, DNA packaging, and genome copy number," Biochimica et Biophysica Acta, vol. 1819, no. 9-10, pp. 921-929, 2012.

[99] K. Kasashima and H. Endo, "Interaction of human mitochondrial transcription factor A in mitochondria: its involvement in the dynamics of mitochondrial DNA nucleoids," Genes to Cells, vol. 20, no. 12, pp. 1017-1027, 2015.

[100] H. B. Ngo, G. A. Lovely, R. Phillips, and D. C. Chan, "Distinct structural features of TFAM drive mitochondrial DNA packaging versus transcriptional activation," Nature Communications, vol. 5, no. 3077, pp. 1-23, 2014.

[101] G. Farge, M. Mehmedovic, M. Baclayon et al., "In vitroreconstituted nucleoids can block mitochondrial DNA replication and transcription," Cell Reports, vol. 8, no. 1, pp. 66-74, 2014.

[102] T. I. Alam, T. Kanki, T. Muta et al., "Human mitochondrial DNA is packaged with TFAM," Nucleic Acids Research, vol. 31, no. 6, pp. 1640-1645, 2003.

[103] K. Kasashima, Y. Nagao, and H. Endo, "Dynamic regulation of mitochondrial genome maintenance in germ cells," Reproductive Medicine and Biology, vol. 13, no. 1, pp. 11-20, 2014.

[104] B. Lu, J. Lee, X. Nie et al., "Phosphorylation of human TFAM in mitochondria impairs DNA binding and promotes degradation by the AAA+ Lon protease," Molecular Cell, vol. 49, no. 1, pp. 121-132, 2013.
[105] A. H. Marceau, "Functions of single-strand DNA-binding proteins in DNA replication, recombination, and repair," Methods in Molecular Biology, vol. 922, pp. 1-21, 2012.

[106] T. Olejar, D. Pajuelo-Reguera, L. Alan, A. Dlaskova, and P. Jezek, "Coupled aggregation of mitochondrial single-strand DNA-binding protein tagged with Eos fluorescent protein visualizes synchronized activity of mitochondrial nucleoids," Molecular Medicine Reports, vol. 12, no. 4, pp. 5185-5190, 2015.

[107] N. Arakaki, T. Nishihama, A. Kohda et al., "Regulation of mitochondrial morphology and cell survival by Mitogenin I and mitochondrial single-stranded DNA binding protein," Biochimica et Biophysica Acta, vol. 1760, no. 9, pp. 13641372, 2006.

[108] J. A. Korhonen, X. H. Pham, M. Pellegrini, and M. Falkenberg, "Reconstitution of a minimal mtDNA replisome in vitro," The EMBO Journal, vol. 23, no. 12, pp. 24232429, 2004.

[109] D. Milenkovic, S. Matic, I. Kuhl et al., "TWINKLE is an essential mitochondrial helicase required for synthesis of nascent D-loop strands and complete mtDNA replication," Human Molecular Genetics, vol. 22, no. 10, pp. 1983-1993, 2013.

[110] S. Gumeni and I. P. Trougakos, "Cross talk of proteostasis and mitostasis in cellular homeodynamics, ageing, and disease," Oxidative Medicine and Cellular Longevity, vol. 2016, Article ID 4587691, 24 pages, 2016.

[111] J. K. Ngo, L. C. Pomatto, and K. J. Davies, "Upregulation of the mitochondrial Lon protease allows adaptation to acute oxidative stress but dysregulation is associated with chronic stress, disease, and aging," Redox Biology, vol. 1, no. 1, pp. 258-264, 2013.

[112] S. Li and D. Rousseau, "ATAD3, a vital membrane bound mitochondrial ATPase involved in tumor progression," Journal of Bioenergetics and Biomembranes, vol. 44, no. 1, pp. 189-197, 2012.

[113] K. Kasashima, M. Sumitani, M. Satoh, and H. Endo, "Human prohibitin 1 maintains the organization and stability of the mitochondrial nucleoids," Experimental Cell Research, vol. 314, no. 5, pp. 988-996, 2008.

[114] M. Kucej, B. Kucejova, R. Subramanian, X. J. Chen, and R. A. Butow, "Mitochondrial nucleoids undergo remodeling in response to metabolic cues," Journal of Cell Science, vol. 121, no. 11, pp. 1861-1868, 2008.

[115] X. J. Chen, X. Wang, B. A. Kaufman, and R. A. Butow, "Aconitase couples metabolic regulation to mitochondrial DNA maintenance," Science, vol. 307 , no. 5710, pp. $714-$ 717, 2005.

[116] J. Kaukonen, J. K. Juselius, V. Tiranti et al., "Role of adenine nucleotide translocator 1 in mtDNA maintenance," Science, vol. 289, no. 5480, pp. 782-785, 2000.

[117] S. Sengupta and C. C. Harris, "p53: traffic cop at the crossroads of DNA repair and recombination," Nature Reviews. Molecular Cell Biology, vol. 6, no. 1, pp. 44-55, 2005.

[118] J. H. Park, J. Zhuang, J. Li, and P. M. Hwang, "p53 as guardian of the mitochondrial genome," FEBS Letters, vol. 590, no. 7, pp. 924-934, 2016.

[119] Y. Yoshida, H. Izumi, T. Torigoe et al., "P53 physically interacts with mitochondrial transcription factor $\mathrm{A}$ and differentially regulates binding to damaged DNA," Cancer Research, vol. 63, no. 13, pp. 3729-3734, 2003. 
[120] K. Heyne, S. Mannebach, E. Wuertz, K. X. Knaup, M. Mahyar-Roemer, and K. Roemer, "Identification of a putative p53 binding sequence within the human mitochondrial genome," FEBS Letters, vol. 578, no. 1-2, pp. 198-202, 2004.

[121] S. Caielli, S. Athale, B. Domic et al., "Oxidized mitochondrial nucleoids released by neutrophils drive type I interferon production in human lupus," The Journal of Experimental Medicine, vol. 213, no. 5, pp. 697-713, 2016.

[122] F. Malka, A. Lombes, and M. Rojo, "Organization, dynamics and transmission of mitochondrial DNA: focus on vertebrate nucleoids," Biochimica et Biophysica Acta, vol. 1763, no. 5-6, pp. 463-472, 2006.

[123] T. Liu, B. Lu, I. Lee, G. Ondrovicova, E. Kutejova, and C. K. Suzuki, "DNA and RNA binding by the mitochondrial Lon protease is regulated by nucleotide and protein substrate," The Journal of Biological Chemistry, vol. 279, no. 14, pp. 13902-13910, 2004.

[124] L. Liu, M. Sanosaka, S. Lei et al., "LRP130 protein remodels mitochondria and stimulates fatty acid oxidation," The Journal of Biological Chemistry, vol. 286, no. 48, pp. 41253-41264, 2011. 


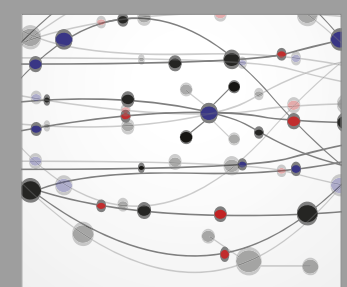

The Scientific World Journal
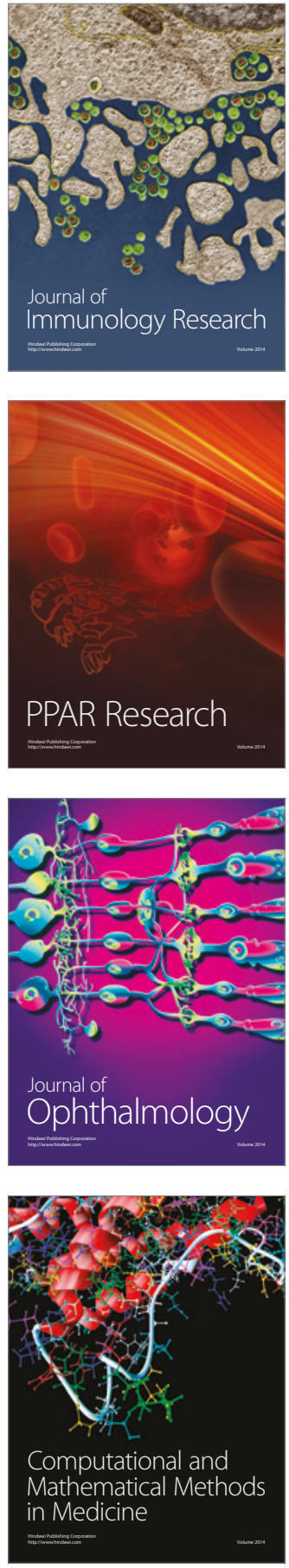

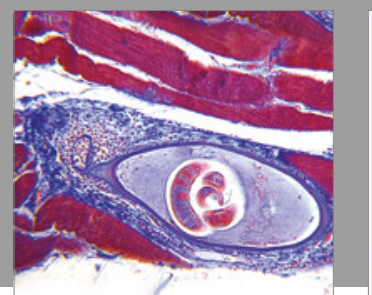

Gastroenterology Research and Practice
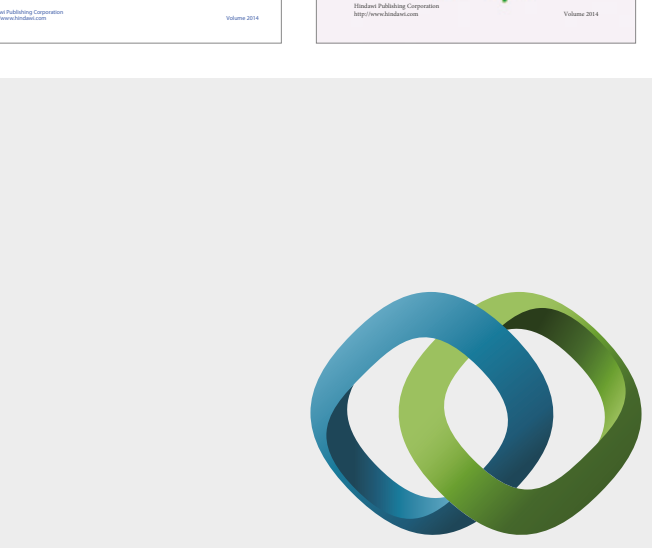

\section{Hindawi}

Submit your manuscripts at

https://www.hindawi.com
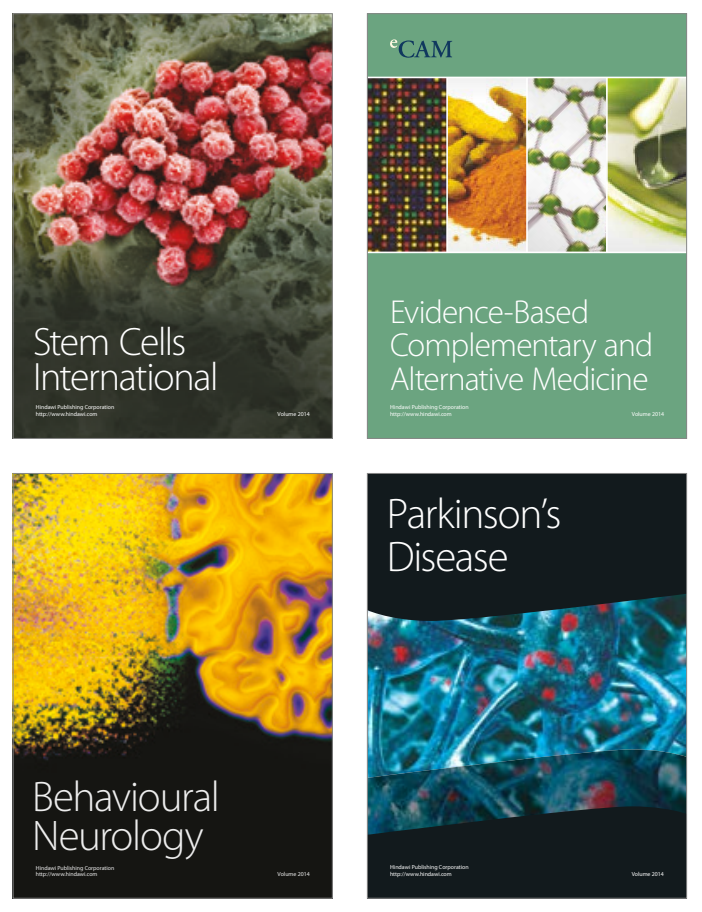
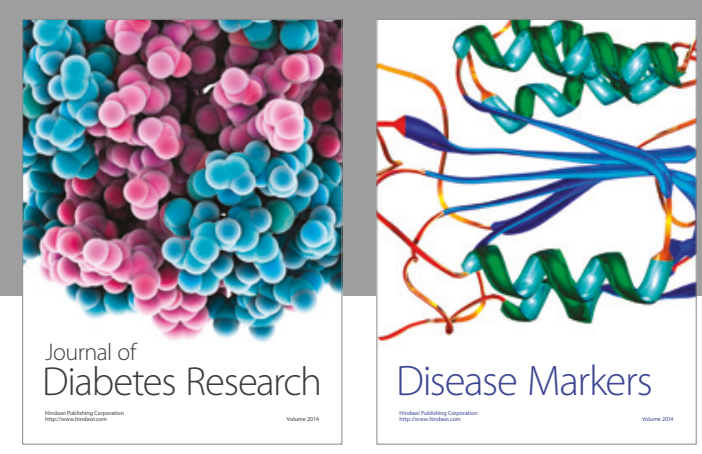

Disease Markers
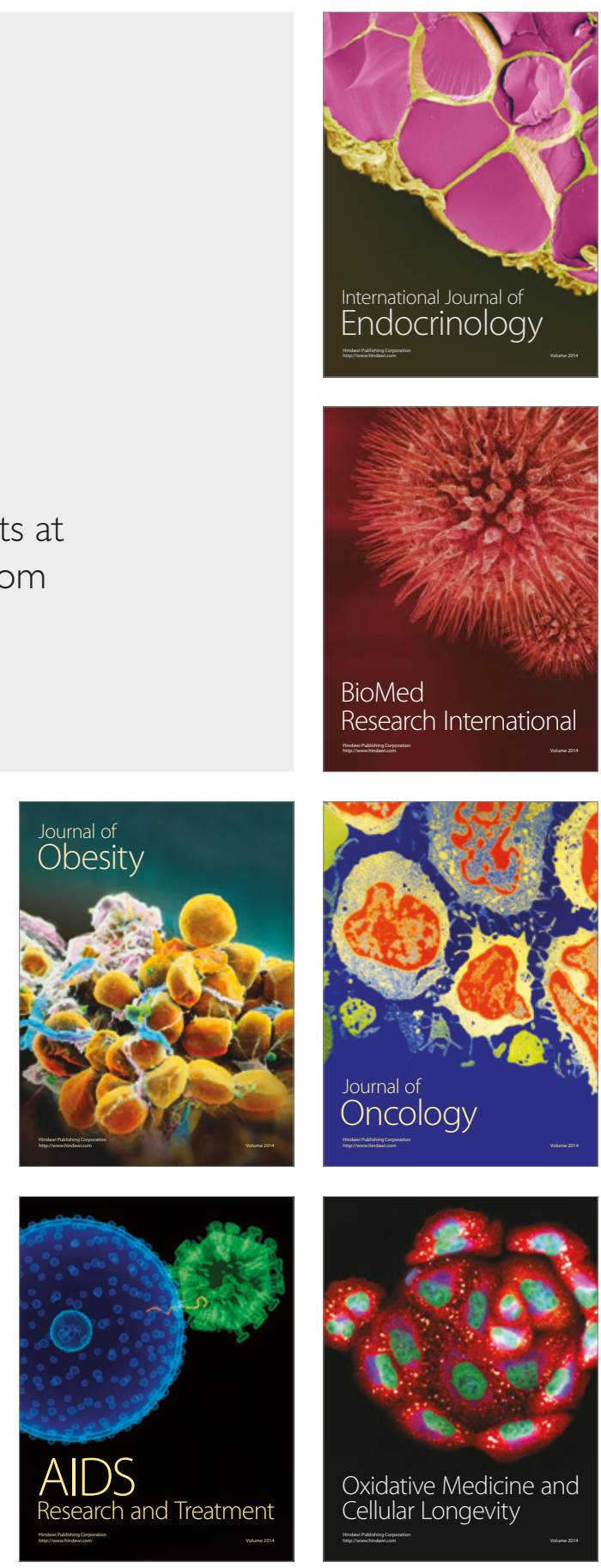